\title{
A Case Study on the Relationship between Fitness Intensity and Dietary Pattern to Intestinal Flora
}

\author{
Xiaoao Zou', Junhui Gao ${ }^{*}$ \\ ${ }^{1}$ The Village School, Houston, USA \\ ${ }^{2}$ American and European International Study Center, Wuxi, China \\ Email: *jhgao68@163.com
}

How to cite this paper: Zou, X.A. and Gao, J.H. (2018) A Case Study on the Relationship between Fitness Intensity and Dietary Pattern to Intestinal Flora. Health, 10, 1037-1043.

https://doi.org/10.4236/health.2018.108078

Received: July 1, 2018

Accepted: August 7, 2018

Published: August 10, 2018

Copyright (c) 2018 by authors and Scientific Research Publishing Inc. This work is licensed under the Creative Commons Attribution International License (CC BY 4.0).

http://creativecommons.org/licenses/by/4.0/

\begin{abstract}
Exercise and diet are two major factors that can cause change to the structure or the biodiversity of human gut microbiota. In this manuscript we have studied relationship between fitness intensity and dietary pattern to intestinal flora by evaluating case studies and analyzing data. We found that if only a single factor was considered, the correlation between diet pattern and individual intestinal flora was higher than that of body strength and individual intestinal flora. If they consider both the correlation between the two and individual intestinal flora, the correlation between fitness intensity was even higher.
\end{abstract}

\section{Keywords}

Exercise Intensity, Diet, Gut Microbiota, Data Analysis

\section{Introduction}

In recent years, human gut microbiota has been discussed by the public because of its health benefits. There are about 100 trillion microbes, including bacterium, fungi, and viruses, found in our gastrointestinal tract (GI tract). They form an ecosystem within our gut. You might wonder why are they allowed to live and reproduce inside our body, instead of being destroyed by our immune system? In fact, human immune system has learnt to live symbiotically with those microbes. Some bacteria in the GI tract are pathogenic. If their number increased sharply or if they enter other parts of the body, the risk of getting inflammation would increase [1]. The immune system will kill bacteria which cross the intestinal barrier [2]. Fortunately, most microbes can help with our digestive sys- 
tem and boost our metabolism. The main food for intestinal microbes is dietary fiber that's difficult to digest. When microbiota breaks down these fibers, they produce short-chain fatty acids, vitamins and amino acids that human body cannot make. Butyrate, a short-chain fatty acid, can repair intestinal mucosal epithelial cells, provide cells with energy, and increase satiety [3]. The loss of short-chain fatty acids is also associated with obesity [4]. In addition, the host's eating habits possibly determine the dominant flora. For example, people who have a high protein diet show a bigger proportion of genus Bacteroides than the general population. But they have fewer bacteria that belong to phylum Firmicutes. On the other side, population who consumes more fibers in their diet shows a greater percentage of genus Prevotella [5]. Prevotella abundance is commonly used as a biomarker for lifestyle and dietary habits in microbiota studies [6].

The growing interest in human gut microbiota has led to the publication of numerous scientific articles, that seek to understand the molecular biology of these microbes and learn how to live in harmony with them. In this paper, we explore the effects of diet and exercise intensity on intestinal flora. We will accomplish our goal by analyzing a study done on professional cyclists. We hope to solve questions like: How would your diet change the composition of your gut microbiota? Does exercise intensity play a role in determining the concentration of intestinal flora? Diet and exercise are two major factors that seem to have an impact on microbes in our GI tract. It's beneficial for our health to understand the interrelationships. It's also exciting to know if we can improve our overall wellbeing by making changes to our daily habits.

\section{Results}

After data analysis, our results are as shown: the first, exercise intensity is related to individual's microbiota composition, the second, diet habit is strongly related to individual's microbiota composition.

\section{Materials and Methods}

\subsection{Data Collection}

We used data from [7]. The table below shows participants' data, including their diet habit, alcohol consumption, exercise load, and race category (recorded from usacycling.com). It also displays Prevotella abundance, mWGS taxonomic cluster.

\subsection{Analyzing the Relationship between Exercise Intensity and Prevotella Abundance}

In Table 1, exercise load is categorized using hours per week. It can be represented by four groups: $6-10,11-15,16-20,20+$. For the sake of simplicity, we will regard $20+$ as $21-25$. Then, we take the average of each work load, simplifying the groups as $8,13,18$, and 23 . In order to obtain Table 2, first we 
Table 1. Reported metadata.

\begin{tabular}{|c|c|c|c|c|c|c|c|}
\hline Cyclist & Sex & Diet & $\begin{array}{c}\text { \# alcohol } \\
\text { beverages per } \\
\text { week }\end{array}$ & $\begin{array}{l}\text { Exercise load } \\
\text { (h/week) }\end{array}$ & $\begin{array}{c}\% \text { abundance } \\
\text { Prevotella (mWGS) }\end{array}$ & $\begin{array}{c}\text { Taxonomiccluster } \\
\text { (mWGS) }\end{array}$ & Race category \\
\hline Knolly & $\mathrm{F}$ & Equal protein, fat, carbs & $1-5$ & $6-10$ & $0.20 \%$ & 3 & PRO \\
\hline Santa Cruz & $\mathrm{F}$ & Equal protein, fat, carbs & 0 & $6-10$ & $0.17 \%$ & 2 & PRO \\
\hline Pivot & $\mathrm{M}$ & Equal protein, fat, carbs & 0 & $6-10$ & $0.13 \%$ & 2 & CAT 1 \\
\hline Breezer & $\mathrm{F}$ & Equal protein, fat, carbs & $6-10$ & $6-10$ & $0.13 \%$ & 2 & PRO \\
\hline Intense & $\mathrm{F}$ & Vegetarian & $1-5$ & $6-10$ & $0.49 \%$ & 2 & CAT 1 \\
\hline Deity & M & Equal protein, fat, carbs & $1-5$ & $6-10$ & $0.13 \%$ & 3 & CAT 1 \\
\hline Renthal & M & Equal protein, fat, carbs & $1-5$ & $6-10$ & $0.20 \%$ & 2 & PRO \\
\hline Iron Horse & M & Equal protein, fat, carbs & $1-5$ & $6-10$ & $0.13 \%$ & 2 & CAT 1 \\
\hline Scott & M & Equal protein, fat, carbs & $1-5$ & $11-15$ & $0.33 \%$ & 2 & PRO \\
\hline Devinci & M & Equal protein, fat, carbs & $1-5$ & $11-15$ & $0.15 \%$ & 3 & PRO \\
\hline Ibis & M & Equal protein, fat, carbs & $1-5$ & $11-15$ & $2.65 \%$ & 3 & PRO \\
\hline Juliana & M & Equal protein, fat, carbs & $1-5$ & $11-15$ & $0.18 \%$ & 2 & PRO \\
\hline Merlin & M & High complex carbs & $1-5$ & $11-15$ & $0.70 \%$ & 3 & PRO \\
\hline Schwinn & M & Paleo & 0 & $11-15$ & $2.35 \%$ & 2 & CAT 1 \\
\hline Mongoose & M & Equal protein, fat, carbs & $1-5$ & $11-15$ & $0.08 \%$ & 3 & PRO \\
\hline Huffy & $\mathrm{F}$ & Paleo & 0 & $11-15$ & $9.02 \%$ & 3 & CAT 1 \\
\hline Giant & M & Equal protein, fat, carbs & 0 & $11-15$ & $1.12 \%$ & 2 & PRO \\
\hline Commencal & M & Paleo & $1-5$ & $11-15$ & $9.93 \%$ & 3 & CAT 1 \\
\hline Cove & $\mathrm{F}$ & Paleo & $1-5$ & $11-15$ & $0.19 \%$ & 2 & PRO \\
\hline Jamis & M & Equal protein, fat, carbs & $15+$ & $11-15$ & $49.11 \%$ & 1 & CAT 1 \\
\hline Yeti & $\mathrm{F}$ & Gluten-free & $1-5$ & $11-15$ & $27.18 \%$ & 3 & PRO \\
\hline Zipp & M & Equal protein, fat, carbs & $1-5$ & $11-15$ & $35.66 \%$ & 1 & PRO \\
\hline Saint & $\mathrm{F}$ & Equal protein, fat, carbs & 0 & $11-15$ & $38.19 \%$ & 1 & PRO \\
\hline Crank & $\mathrm{F}$ & Paleo & 0 & $11-15$ & $14.67 \%$ & 1 & PRO \\
\hline Pinarello & M & Equal protein, fat, carbs & 0 & $11-15$ & $45.27 \%$ & 1 & CAT 1 \\
\hline Trek & M & Equal protein, fat, carbs & $1-5$ & $16-20$ & $49.52 \%$ & 1 & CAT 1 \\
\hline Niner & $\mathrm{F}$ & Paleo & $1-5$ & $16-20$ & $0.36 \%$ & 2 & CAT 1 \\
\hline Norco & M & High complex carbs & $6-10$ & $16-20$ & $38.47 \%$ & 1 & PRO \\
\hline Enve & M & Equal protein, fat, carbs & $1-5$ & $16-20$ & $14.74 \%$ & 3 & PRO \\
\hline Speed Play & M & Equal protein, fat, carbs & $1-5$ & $16-20$ & $10.53 \%$ & 3 & PRO \\
\hline SRAM & M & Equal protein, fat, carbs & $1-5$ & $20+$ & $7.53 \%$ & 3 & PRO \\
\hline Easton & M & High complex carbs & $1-5$ & $20+$ & $27.03 \%$ & 1 & PRO \\
\hline Thomson & $\mathrm{F}$ & Gluten-free & 0 & $20+$ & $12.12 \%$ & 3 & PRO \\
\hline
\end{tabular}


Table 2. Exercise load/intensity and Prevotella abundance.

\begin{tabular}{ccccc}
\hline & 8 & 13 & 18 & 23 \\
\hline CAT & 0.022 & 0.23136 & 0.2494 & \\
PRO & 0.00175 & 0.100917 & 0.212467 & 0.1556 \\
\hline
\end{tabular}

divide the athletes by their race category. Second step is calculating the mean Prevotella abundance of each group.

As we can see from Table 2, there isn't a number representing Pro athletes who work 23 hours a week. So we referred to data from the other 3 groups in Pro category and we calculated the number that's missing. We obtained Table 3 by adding a row that shows an average of Cat and Pro.

Figure 1 is obtained by performing a linear regression on Table 3.

From Figure 1, we can see $y$ is $0.0635 x-0.006$ while $\mathrm{R}$ square is 0.7058 .

\subsection{Diet and Prevotella Abundance Correlation}

In Table 1, there're 5 eating habits shown. They are vegetarian, Paleo, Equal protein-fat-carbs, gluten free, and high complex carbs. Table 4 is obtained by calculating the mean value of Prevotella abundance (mWGS).

Figure 2 is obtained by performing linear regression on the data shown in Table 4.

In Table 4, $\mathrm{Y}$-axis represents average Prevotella abundance and $\mathrm{X}$-axis represents types of diet. We can get the linear equation which is $y=0.0113 x-$ 0.0492 , the coefficient of determination being 0.9828 .

\subsection{Considering Two Factors Together}

In 3.2, we investigated the relationship between exercise intensity and Prevotella abundance. In 3.3, we investigated the relationship between diet and Prevotella abundance. From the coefficient R squared, dietary patterns are more closely related to intestinal flora.

Tables 5-7 below are obtained by performing a multiple linear regression on both factors.

Analysis: When we are considering both factors(diet and exercise intensity) as independent variables, the coefficient of determination is 0.19 . However, when we look back to the individual regression done on each factor, the coefficients were closer to 1 . From the standard error, we see the average error of estimation is off by 15.35 which is relatively high. In this multiple linear regression, both diet and exercise load are in positive relationship with Prevotella abundance. Diet shows a stronger link compared to exercise load. We could assume that a change in diet effect the abundance of particular gut microbiota in a greater way than changing exercise load.

\section{Discussion and Conclusion}

There are certainly other factors that can affect the abundance of different gut 
Table 3. Exercise load/intensity and Prevotella abundance (missing value filled in).

\begin{tabular}{ccccc}
\hline & 8 & 13 & 18 & 23 \\
\hline CAT & 0.022 & 0.23136 & 0.2494 & 0.2482 \\
PRO & 0.00175 & 0.100917 & 0.212467 & 0.1556 \\
Avg & 0.011875 & 0.166139 & 0.230934 & 0.2019 \\
\hline
\end{tabular}

Table 4. Diet and Prevotella abundance.

\begin{tabular}{ccc}
\hline Diet & Degree & mWGS \\
\hline Vegetarian & 5 & 0.0049 \\
Paleo & 10 & 0.060866665 \\
Equal-protein-fat-carbs & 15 & 0.121976191 \\
Gluten-free & 20 & 0.196500007 \\
High complex carbs & 25 & 0.220666667 \\
\hline
\end{tabular}

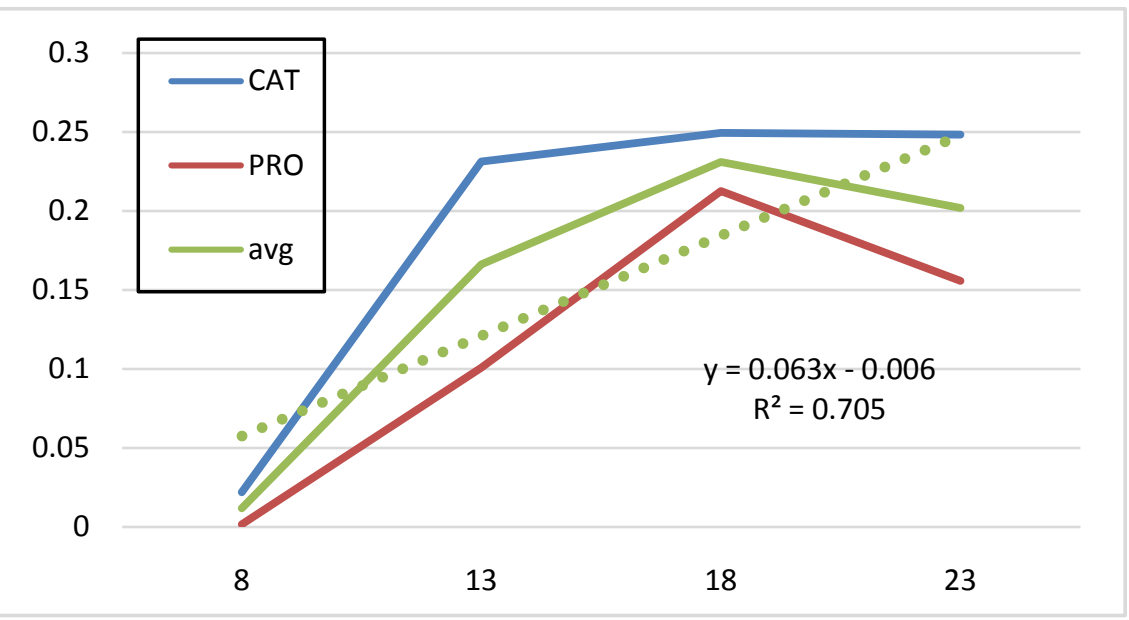

Figure 1. Relationship between exercise intensity and Prevotella abundance.

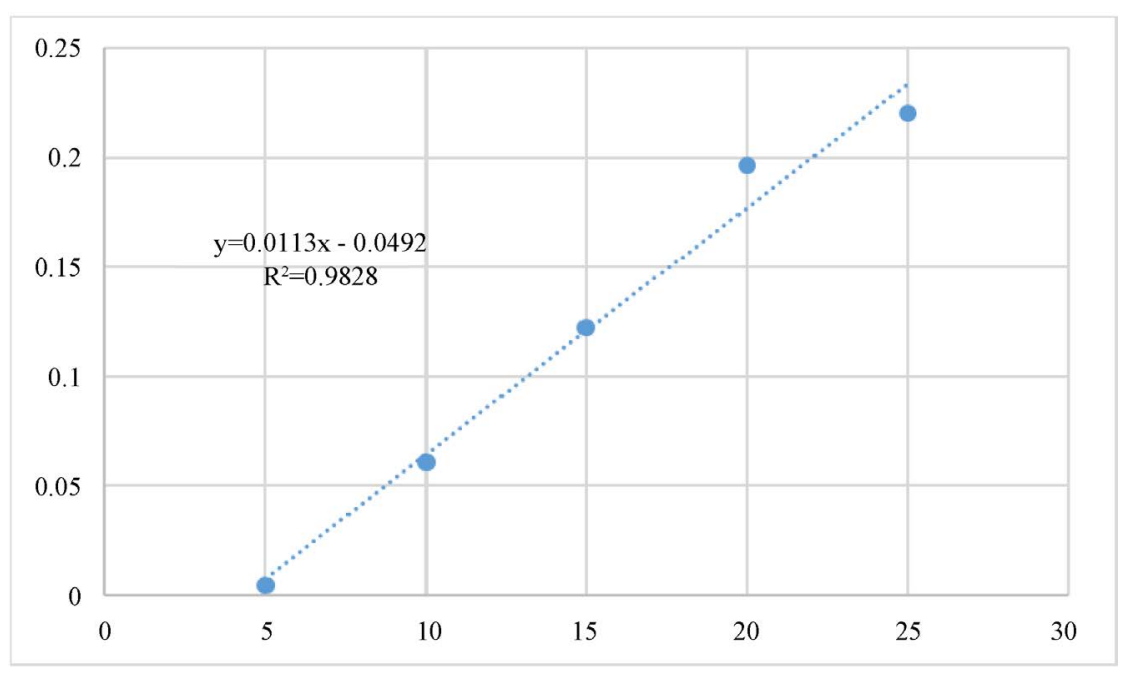

Figure 2. Correlation between diet and Prevotella abundance. 
Table 5. Regression statistics.

\begin{tabular}{cc}
\hline Multiple R & 0.435321621 \\
R Square & 0.189504914 \\
Adjusted R Square & 0.135471908 \\
Standard Error & 15.34762221 \\
Observations & 33 \\
\hline
\end{tabular}

Table 6. ANOVA

\begin{tabular}{cccccc}
\hline & df & SS & MS & F & Significance F \\
\hline Regression & 2 & 1652.241567 & 826.1207834 & 3.507206585 & 0.042781479 \\
Residual & 30 & 7066.485221 & 235.5495074 & & \\
Total & 32 & 8718.726788 & & & \\
\hline
\end{tabular}

Table 7. Coefficient table.

\begin{tabular}{ccccc}
\hline & Coefficients & Standard Error & t Stat & P-value \\
\hline Intercept & -17.46333043 & 11.74965846 & -1.48628409 & 0.1476387 \\
diet & 3.385515337 & 3.329533079 & 1.016813846 & 0.317372703 \\
Exercise load & 1.470767553 & 0.751658231 & 1.956697197 & 0.059747245 \\
\hline
\end{tabular}

flora. However, we were not able to further investigate due to the lack of data. What's more, our article needs more case studies to be convincible.

In this article, we built mathematical models to analyze data obtained from a case study. Then we calculated and investigated on the effects of exercise intensity and diet on gut microbiota.

\section{Conflicts of Interest}

The authors declare no conflicts of interest regarding the publication of this paper.

\section{References}

[1] Hooper, L.V. and Macpherson, A.J. (2010) Immune Adaptations that Maintain Homeostasis with the Intestinal Microbiota. Advances in Pediatrics, US National Library of Medicine, March 2010.

[2] Hamer, H.M., et al. (2008) Review Article: The Role of Butyrate on Colonic Function. Advances in Pediatrics, US National Library of Medicine, 15 January 2008.

[3] Li, X., et al. (2017) Advances in Pediatrics, US National Library of Medicine.

[4] Wei, H. and Duan, L.P. (2017) Research Progress on the Influence of Diet on the Structure, Metabolism and Function of Intestinal Flora. Chinese Journal of Digestion, 9.

[5] De Filippo, C., Cavalieri, D., Di Paola, M., Ramazzotti, M., Poullet, J.B., Massart, S., Collini, S., Pieraccini, G. and Lionetti, P. (2010) Impact of Diet in Shaping Gut Microbiota Revealed by a Comparative Study in Children from Europe and Rural 
Africa. Proceedings of the National Academy of Sciences of the United States of America, 107, 14691-14696. https://doi.org/10.1073/pnas.1005963107

[6] Gorvitovskaia, A., et al. (2016) Interpreting Prevotella and Bacteroides as Biomarkers of Diet and Lifestyle. Microbiome, BioMed Central, 12 April 2016.

[7] Petersen, L.M., Bautista, E.J., Nguyen, H., Hanson, B.M., Chen, L., Lek, S.H., Sodergren, E. and Weinstock, G.M. (2017) Community Characteristics of the Gut Microbiomes of Competitive Cyclists. Microbiome, 5, 98. 